\title{
MULTISEASONAL TREE CROWN STRUCTURE MAPPING WITH POINT CLOUDS FROM OTS QUADROCOPTER SYSTEMS
}

\author{
S. Hese ${ }^{\mathrm{a} *}$ F. Behrendt ${ }^{\mathrm{a}}$ \\ ${ }^{a}$ Friedrich-Schiller-University Jena, Earth Observation Department, Institute for Geography, 07743 Jena, Germany - \\ soeren.hese@uni-jena.de
}

Commission VI, WG VI/4

KEY WORDS: Tree Crown Structure, Point Cloud, Quadrocopter, OTS, Point Density, OBIA, Multi Seasonal

\begin{abstract}
:
OTF (Off The Shelf) quadro copter systems provide a cost effective (below 2000 Euro), flexible and mobile platform for high resolution point cloud mapping. Various studies showed the full potential of these small and flexible platforms. Especially in very tight and complex 3D environments the automatic obstacle avoidance, low copter weight, long flight times and precise maneuvering are important advantages of these small OTS systems in comparison with larger octocopter systems. This study examines the potential of the DJI Phantom 4 pro series and the Phantom 3A series for within-stand and forest tree crown 3D point cloud mapping using both within stand oblique imaging in different altitude levels and data captured from a nadir perspective. On a test site in Brandenburg/Germany a beach crown was selected and measured with 3 different altitude levels in Point Of Interest (POI) mode with oblique data capturing and deriving one nadir mosaic created with 85/85\% overlap using Drone Deploy automatic mapping software. Three different flight campaigns were performed, one in September 2016 (leaf-on), one in March 2017 (leaf-off) and one in May 2017 (leaf-on) to derive point clouds from different crown structure and phenological situations - covering the leaf-on and leafoff status of the tree crown. After height correction, the point clouds where used with GPS geo referencing to calculate voxel based densities on $50 \times 10 \times 10 \mathrm{~cm}$ voxel definitions using a topological network of chessboard image objects in $0,5 \mathrm{~m}$ height steps in an object based image processing environment. Comparison between leaf-off and leaf-on status was done on volume pixel definitions comparing the attributed point densities per volume and plotting the resulting values as a function of distance to the crown center. In the leaf-off status SFM (structure from motion) algorithms clearly identified the central stem and also secondary branch systems. While the penetration into the crown structure is limited in the leaf-on status (the point cloud is a mainly a description of the interpolated crown surface) - the visibility of the internal crown structure in leaf-off status allows to map also the internal tree structure up to and stopping at the secondary branch level system. When combined the leaf-on and leaf-off point clouds generate a comprehensive tree crown structure description that allows a low cost and detailed 3D crown structure mapping and potentially precise biomass mapping and/or internal structural differentiation of deciduous tree species types. Compared to TLS (Terrestrial Laser Scanning) based measurements the costs are neglectable and in the range of 1500-2500€. This suggests the approach for low cost but fine scale in-situ applications and/or projects where TLS measurements cannot be derived and for less dense forest stands where POI flights can be performed. This study used the in-copter GPS measurements for geo referencing. Better absolute geo referencing results will be obtained with DGPS reference points. The study however clearly demonstrates the potential of OTS very low cost copter systems and the image attributed GPS measurements of the copter for the automatic calculation of complex 3D point clouds in a multi temporal tree crown mapping context.
\end{abstract}

\section{INTRODUCTION}

Small Off The Shelf (OTS) quadro copter systems provide a very cost effective, highly flexible, mobile platform for high resolution point cloud mapping. Various studies showed the full potential of these small and flexible platforms (f.e. Mlambo et al. 2017). An overview is given in Colomina and Molina (2014). Especially in very complex 3D environments the automatic obstacle avoidance, low copter weight, long flight times and precise maneuvering is an important advantage of small OTS systems in comparison with larger octocopter systems. Especially for the mapping of $2.5 \mathrm{D}$ structures mapping a continuous height surface of a stand and not 3D structures - the airborne Lidar and UAV SfM approaches creates comparable surface representation results (Thiel \& Schmullius 2016). In comparison with airborne Lidar scanning and terrestrial Lidar point data capturing methods the penetration of the point cloud from overlapping images is however clearly limited. This has already been demonstrated in Wallace et al. 2016. However, in combination with leaf-off data the SfM approach might generate a much denser point cloud that also reproduces the internal structure of a tree crown. SfM algorithms clearly can generate similar high quality $3 \mathrm{D}$ point clouds as from Aerial Laser Scanning (ALS). This has been demonstrated in various studies as published f.e. in Fonstad et al (2013), Javernick et al. (2014) and Mancini et al. (2013). Since the development of very fast Graphics Processing Units (GPU) with multiple processing cores the computing times for image matching have decreased considerably making high quality matching projects with thousands of 20 megapixel image files possible now.

This study examines the potential of the DJI Phantom 4 Pro series images and the Phantom 3 Advanced (Figure 1) images for within stand and forest tree crown 3D point cloud mapping

* Corresponding author 
using both within stand oblique imaging in different altitude levels and data capturing from a nadir perspective.

\section{DATA AND STUDY AREA}

On a test site in Brandenburg/Germany a beech crown was selected and measured with 3 different altitude levels in Point Of Interest (POI) flight mode with oblique data capturing and deriving one nadir mosaic created with $85 / 85 \%$ overlap using Drone Deploy automatic mapping software. Data was captured in a raw image file format, as 20 megapixel DNG (Adobe Digital Negative format) image files with a $24 \mathrm{~mm}$ equiv. focal length (Phantom 4 Pro) and with 12 megapixel DNG image files for the Phantom 3A (Figure 1) with a $20 \mathrm{~mm}$ focal length equivalent. Pre-processing of image files was performed using Adobe Lightroom software applying chromatic aberration correction, defringing, noise reduction and masked sharpening for batch output to uncompressed - quasi lossless JPEG format - with 20 megapixel resolution. DJI Phantom 3 A image files were corrected using the Adobe provided lens correction profiles for the FC300S camera system. For the Phantom 4 Pro lens/camera system a manual correction profile was applied. Three different flight campaigns were performed. One in September 2016 (leaf-on) und one in March 2017 (leaf-off) and one in May 2017 (leaf-on) to derive point clouds from different crown structures and leaf density situations - covering the leafon and leaf-off status of the tree crown. 356-443 DNG raw images were exported from every flight campaign including GPS coordinates and relative copter altitude information.

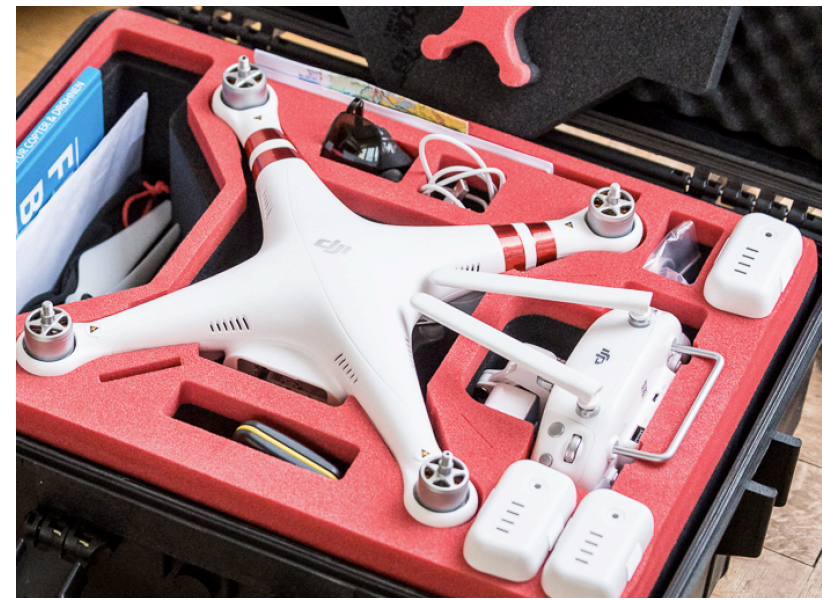

Figure 1. DJI "Phantom 3 Advanced" OTS quadro copter system in the MC case system.

\section{METHODS AND RESULTS}

Image alignment with indirect pitch/roll/aspect calculations and matching to a dense point cloud with approx. 40-50 million points from every flight campaign was done using Agisoft Photoscan software with full resolution image matching and mild depth filtering (leaf-on status from may 2017: 443 images, RMS projection error 0.857 pixel, adaptive camera model fitting allowed, dense point cloud with 39,75 million points, Agisoft reconstruction quality "high" and depth filtering set to "mild", leaf-off status from March 2017: 356 images, RMS projection error 0,506 pixel, 51 million points). Relative geometric accuracy (deviation of the geometric correction from GPS coordinate positions) is given in Figure 2 for the May 2017 flight campaign.

The point cloud (Figure 3) was exported to the las file format and re-projected to UTM33N WGS84. The height information of the point clouds was corrected and normalized manually to a stand height model using mean terrain height values using the LASTools software. The DJI Phantom calculated height information is based on relative barometric information always relative to the start-altitude of the UAV and is not related to geo-referenced height or above-ellipsoid height values.

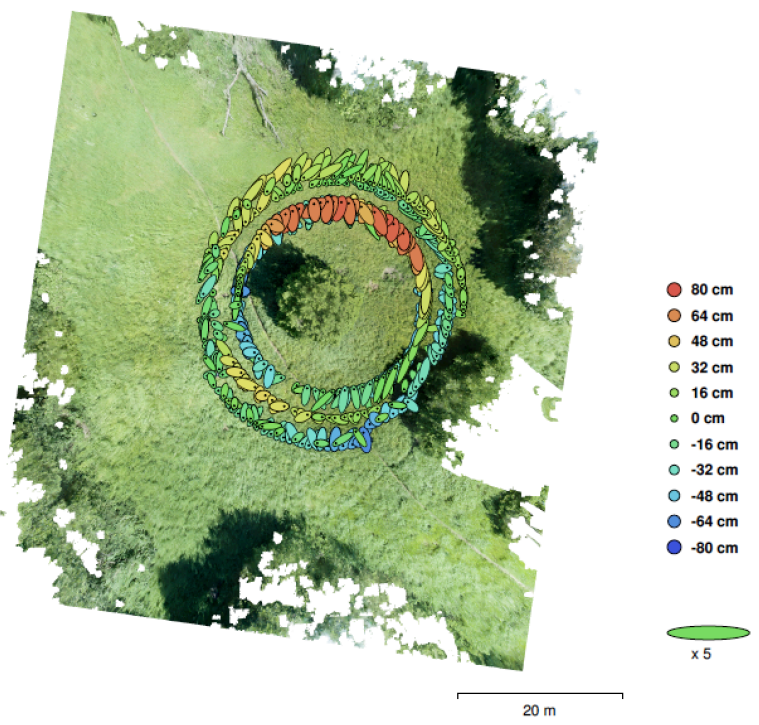

Figure 2. Image locations and relative error estimates in $\mathrm{x} / \mathrm{y}$ directions measured for the POI image locations in three different height levels.

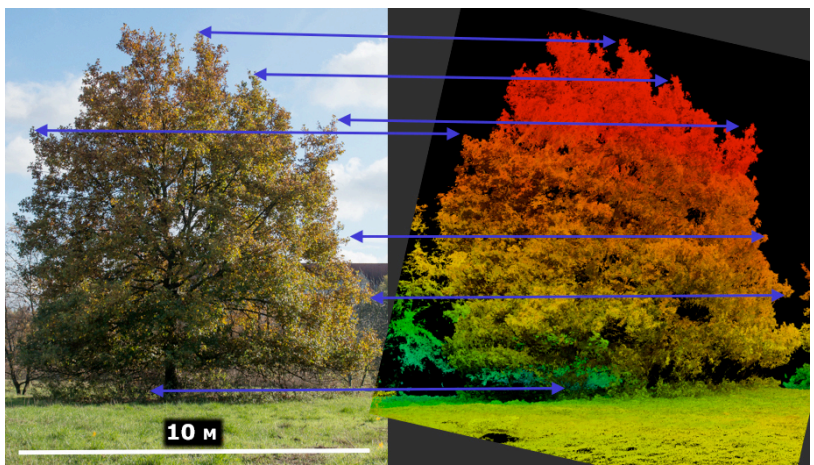

Figure 3. Tree crown structure point cloud (right) in Leaf-On status compared with a horizontal DSLR photograph made from the same perspective (left).

In leaf-off status SfM (structure from motion) algorithms clearly identified the central stem and also secondary branching systems. While the penetration into the crown structure is limited in the leaf-on status (the point cloud is clearly more a description of the crown surface) - in leaf-off status mapping the internal tree structure up to and mainly stopping at the secondary branching system is possible. After height correction, the point clouds where used to calculate pixel volume based densities on $50 \mathrm{~cm}$ (height) x $10 \mathrm{~cm}(\mathrm{x})$ x $10 \mathrm{~cm}$ (y) volume pixel definitions using a topological network of chessboard image objects in $0,5 \mathrm{~m}$ height steps in an object based image processing environment and attributing the densities to chess board image objects. Comparison between leaf-off and leaf-on status was done on volume pixel definitions comparing the attributed point densities per volume and mapping the results in distance to the approximated central stem position. The resulting point density statistics are created for the leaf-on and 
leaf-off status as mean values for different distances to the crown center in various crown height levels.

Results are given in Figure 4-6 for the height levels 6 - 6,5 m meters, 3,5 - 4 meters and 1 - 1,5 meters. The density distribution clearly shows high densities for the leaf-on status in the outer branch system and severe loss of structural description only after approx. $3 \mathrm{~m}$ inwards towards the center. This is visible in all height levels of the leaf-on point cloud model. In comparison, the leaf-off model starts to show higher point densities only in a distance of $0-2,5$ meters to the central stem position indicating a gap in the volume description between 2,5 and $3,5 \mathrm{~m}$ distance to the central stem position. As the density measurement was done as a mean value for all equal radius distance values the directional structural in-homogeneities of the crown system could distort the results but overall the relative density differences are clearly adjoining to the visual point cloud model density analysis. The height level 3.5-4 meters is the only height level that clearly shows a dense point distribution within the full 3D crown space with a slight overlap of leaf-on and leaf-off point cloud model (Figure 5).

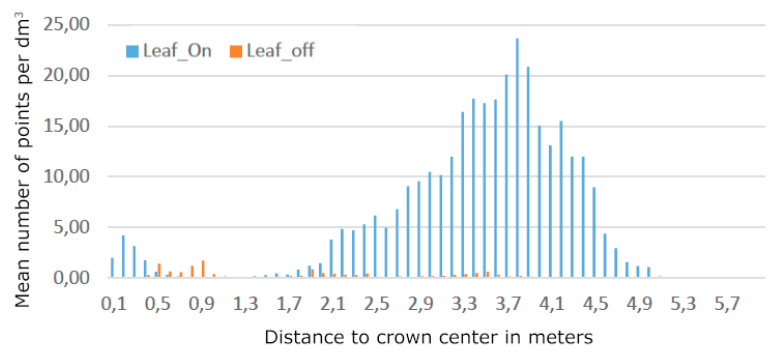

Figure 4. Mean point density per $5 \times 1 \mathrm{dm}^{3}$ in various distances to the crown centre in level $6-6,5 \mathrm{~m}$ height.

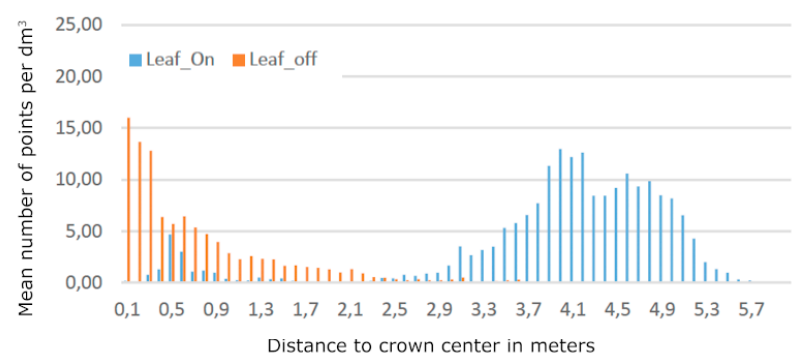

Figure 5. Mean point density per $5 \times 1 \mathrm{dm}^{3}$ in various distances to the crown centre in level 3,5-4 m height.

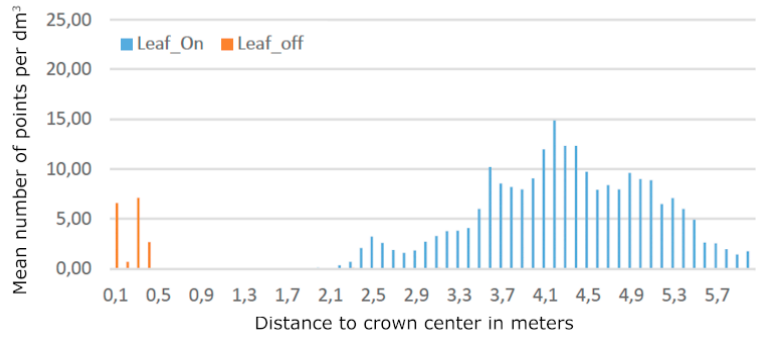

Figure 6 . Mean point density per $5 \times 1 \mathrm{dm}^{3}$ in various distances to the crown centre in level $1-1,5 \mathrm{~m}$ height.

\section{CONCLUSIONS}

When combined, leaf-on and leaf-off point clouds generate a comprehensive tree crown structure description that allows a low cost and detailed 3D crown structure mapping and potentially precise biomass mapping or internal structural differentiation of deciduous tree species tree types (tested in this work with an individual beech tree). Compared to TLS (Terrestrial Laser Scanning) based measurements the costs are neglectable. This suggests the approach for low cost but fine scale in-situ applications and/or projects where TLS measurements cannot be derived. This study used the in-copter GPS measurements for geo-referencing. Better absolute geo referencing results will be obtained with DGPS reference points and better relative fit of the two (leaf-on/off) point cloud models is expected as a consequence. The study however clearly demonstrates the potential of OTS very low cost copter systems and the image attributed GPS measurements of the copter for the automatic calculation of georeferenced complex 3D point cloud models in a multi temporal tree crown leaf-off/leaf-on point mapping context. We did not validate the overall accuracy of the point clouds but visual comparison with 36 megapixel DSLR image mosaics shot horizontally clearly indicates a good fit and high precision of the outer surface description of the leaf-on crown surface and the branch system mapping in a leafoff situation.

\section{ACKNOWLEDGEMENTS}

Acknowledged is the financial support of the Friedrich-SchillerUniversity for development and acquisition of UAV instruments and sensors at Jena Copter Labs (http://wwwjenacopterlabs.de) at the Department for Earth observation, Institute of Geography.

\section{REFERENCES}

Colomina, I., Molina, P., 2014. Unmanned aerial systems for photogrammetry and remote sensing: A review, ISPRS Journal of Photogrammetry and Remote Sensing, Vol. 92, June 2014, p79-97.

Fonstad, M.A.; Dietrich, J.T.; Courville, B.C.; Jensen, J.L.; Carbonneau, P.E. 2013. Topographic Structure from Motion: A New Development in Photogrammetric Measurement. Earth Surf. Process. Landf. 2013, 38, 421-430.

Javernick, L.; Brasington, J.; Caruso, B. 2014. Modeling the Topography of Shallow Braided Rivers Using Structure-fromMotion Photogrammetry. Geomorphology 2014, 213, 166-182.

Mlambo, R.; Woodhouse, I. H.; Gerard, F., Anderson, K., 2017. Structure from Motion (SfM) Photogrammetry with Drone Data: A Low Cost Method for Monitoring Greenhouse Gas Emissions from Forests in Developing Countries. Forests 8, no. 3: 68 .

Mancini, F.; Dubbini, M.; Gattelli, M.; Stecchi, F.; Fabbri, S.; Gabbianelli, G. 2013. Using Unmanned Aerial Vehicles (UAV) for High-Resolution Reconstruction of Topography: The Structure from Motion Approach on Coastal Environments. Remote Sens. 2013, 5, 6880-6898.

Thiel, C., Schmullius, C., 2016. Comparison of UAV photograph-based and airborne lidar-based point clouds over forest from a forestry application perspective, International Journal of Remote Sensing, DOI: 10.1080/01431161.2016.1225181.

Wallace, L., Lucieer, A., Malenovský, Z., Turner, D., Vopěnka, P., 2016. Assessment of Forest Structure Using Two UAV Techniques: A Comparison of Airborne Laser Scanning and Structure from Motion (SfM) Point Clouds. Forests 7, no. 3: 62. 